Article

\title{
Fabrication of Hypericin Imprinted Polymer Nanospheres via Thiol-Yne Click Reaction
}

\author{
Yuxin Pei *, Fengfeng Fan, Xinxin Wang (D), Weiwei Feng, Yong Hou and Zhichao Pei * \\ Shanxi Key Laboratory of Natural Products \& Chemical Biology, College of Chemistry \& Pharmacy, \\ Northwest A\&F University, Yangling 712100, Shaanxi, China; fanfengfeng1314@163.com (F.F.); \\ xinxinwang@nwafu.edu.cn (X.W.); fengweiwei@nwafu.edu.cn (W.F.); houyong2012@126.com (Y.H.) \\ * Correspondence: peiyx@nwafu.edu.cn (Y.P.); peizc@nwafu.edu.cn (Z.P.); Tel.: +86-29-8709-1196 (Y.P. \& Z.P.); \\ Fax: +86-29-8709-2769 (Y.P. \& Z.P.)
}

Received: 31 August 2017; Accepted: 21 September 2017; Published: 24 September 2017

\begin{abstract}
To fabricate molecularly imprinted polymer nanospheres via click reaction, five different clickable compounds were synthesized and two types of click reactions (azide-alkyne and thiol-yne) were explored. It was found that molecularly imprinted polymer nanospheres could be successfully synthesized via thiol-yne click reaction using 3,5-diethynyl-pyridine (1) as the monomer, tris(3-mercaptopropionate) (tri-thiol, 5) as the crosslinker, and hypericin as the template (MIP-NSHs). The click polymerization completed in merely $4 \mathrm{~h}$ to produce the desired MIP-NSHs, which were characterized by FTIR, SEM, DLS, and BET, respectively. The reaction conditions for adsorption capacity and selectivity towards hypericin were optimized, and the MIP-NSHs synthesized under the optimized conditions showed a high adsorption capacity $\left(Q=6.03 \mu \mathrm{mol} \cdot \mathrm{g}^{-1}\right)$ towards hypericin. The imprinting factors of MIP-NSHs towards hypericin, protohypericin, and emodin were 2.44, 2.88, and 2.10 , respectively.
\end{abstract}

Keywords: molecular imprinting; hypericin; click reaction; nanospheres

\section{Introduction}

As an important type of artificial receptor, molecularly imprinted polymers (MIPs) [1,2], proposed in the latter part of the last century, have become increasingly attractive to both the academic community and in industry due to their high affinity towards given molecules (templates), high chemical stability, and low cost [3], and have been applied widely in chromatography [4], solid phase extraction (SPE) [5], separation [6], immunoassay [7], sensors [8], catalysis [9], and drug delivery [10-12]. MIPs are synthesized via the copolymerization of functional monomers and crosslinkers in the presence of template molecules that form assemblies with functional monomers driven by covalent or non-covalent interactions. Sequential removal of the template molecules from the crosslinked polymer networks leaves the recognition cavities complementary to the shape, size, and position of the functional groups, which then show specific recognition and rebinding capacity towards the template molecules upon re-exposure [13].

The most common physical form of MIPs is polymer monolith obtained via bulk polymerization, which usually produces irregular polymeric particles in a range of 5-100 $\mu \mathrm{m}$ in low yield (less than $50 \%)[13,14]$ through a tedious process of crushing, grinding and sieving. Although the method is simple, it causes considerable wastage of the MIPs. More importantly, the irregularities of the resultant particles, in terms of size, shape, and architecture, produced by this method make sample handling difficult and decrease separation efficiency, which limit the application of MIPs, particularly in SPE or chromatography [15-17]. In contrast, spherical particles with a well-defined structure and monodispersity are more desirable due to their fast mass transfer rate, better separation performance 
and low backpressure as solid phase in chromatography column $[18,19]$. To this end, new synthetic methodologies have been important research topics in the past two decades [13]. Consequently, several methods have been successfully established for the fabrication of spherical MIPs beads, including inverse suspension polymerization [20], emulsion polymerization [21], solution polymerization [22], multi-step swelling polymerization [23], and precipitation polymerization [13,24]. However, these methods are either complicated in manipulation, require a long time to complete polymerization, or require surfactants (which are difficult to remove from MIPs) to prevent aggregation of polymer particles formed in the process of polymerization. Development of new methods that can produce MIP nanospheres efficiently with well-defined architecture in a high yield is therefore still much desired.

Click reaction, introduced by Sharpless in 2001 [25], has been recognized as a popular and powerful tool in controlling macromolecular architecture due to its mild, fast, highly efficient and specific reaction characteristics [26]. However, click reaction was not applied in the field of molecular imprinting technology until 2011, when Ye and his co-workers [27] prepared clickable molecularly imprinted core-shell nanoparticles using a simple one-pot precipitation polymerization with sequential addition of monomers containing clickable functional groups. To this day, few reports of click reactions for the synthesis of MIPs have been published in the literature. Examples of this include Xu et al. who prepared 2D molecularly imprinted materials based on mesoporous silicas via click reaction [28], and Mendes et al. who fabricated glycoprotein selective surfaces by using the click-imprinting technique [29]. Extensive application of click reactions in the fabrication of MIPs might be restricted by the shortage of commercially available clickable monomers and crosslinkers.

Previously, with ultrasonic assistance, our group developed a one-step approach to synthesize polymeric nanospheres via click reaction between azide and alkyne $[30,31]$ in the absence of surfactants. The polymerization was completed in $4 \mathrm{~h}$ and the size of the nanospheres were controlled by varying the combination of monomers and crosslinkers and reaction conditions. Meanwhile, hypericin is a photosensitive antiviral, anticancer and antidepressant agent derived from Hypericum perforatum (St John's wort) by multistep extraction, therefore, the development and application of hypericin imprinted polymer can speed up and improve the isolation process or can be used in a sensor for hypercin monitoring in biological samples [32]. In this work, we explored the possibility of using click reaction to synthesize molecularly imprinted nanospheres with hypericin as the template. To our delight, molecularly imprinted nanospheres towards hypericin (MIP-NSHs) were successfully obtained by using 3,5-diethynyl-pyridine (1) as the functional monomer, trimethylolpropane tris(3-mercaptopropionate) (tri-thiol, 5) as the crosslinker in the presence of hypericin (see Scheme 1). The thiol-yne click polymerization only took a mere $4 \mathrm{~h}$ to yield the desired MIP-NSHs. The MIP-NSHs prepared under the optimized conditions displayed a high adsorption capacity $\left(Q=6.03 \mu \mathrm{mol} \cdot \mathrm{g}^{-1}\right)$ and fair selectivity towards hypericin. This work presents a straightforward method to fabricate molecularly imprinted nanospheres via thiol-yne click reaction. 
a)

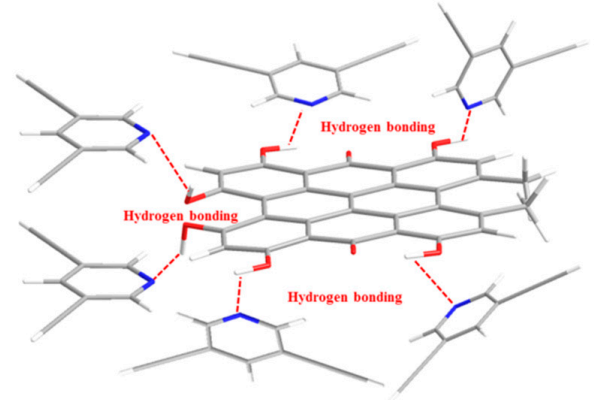

b)

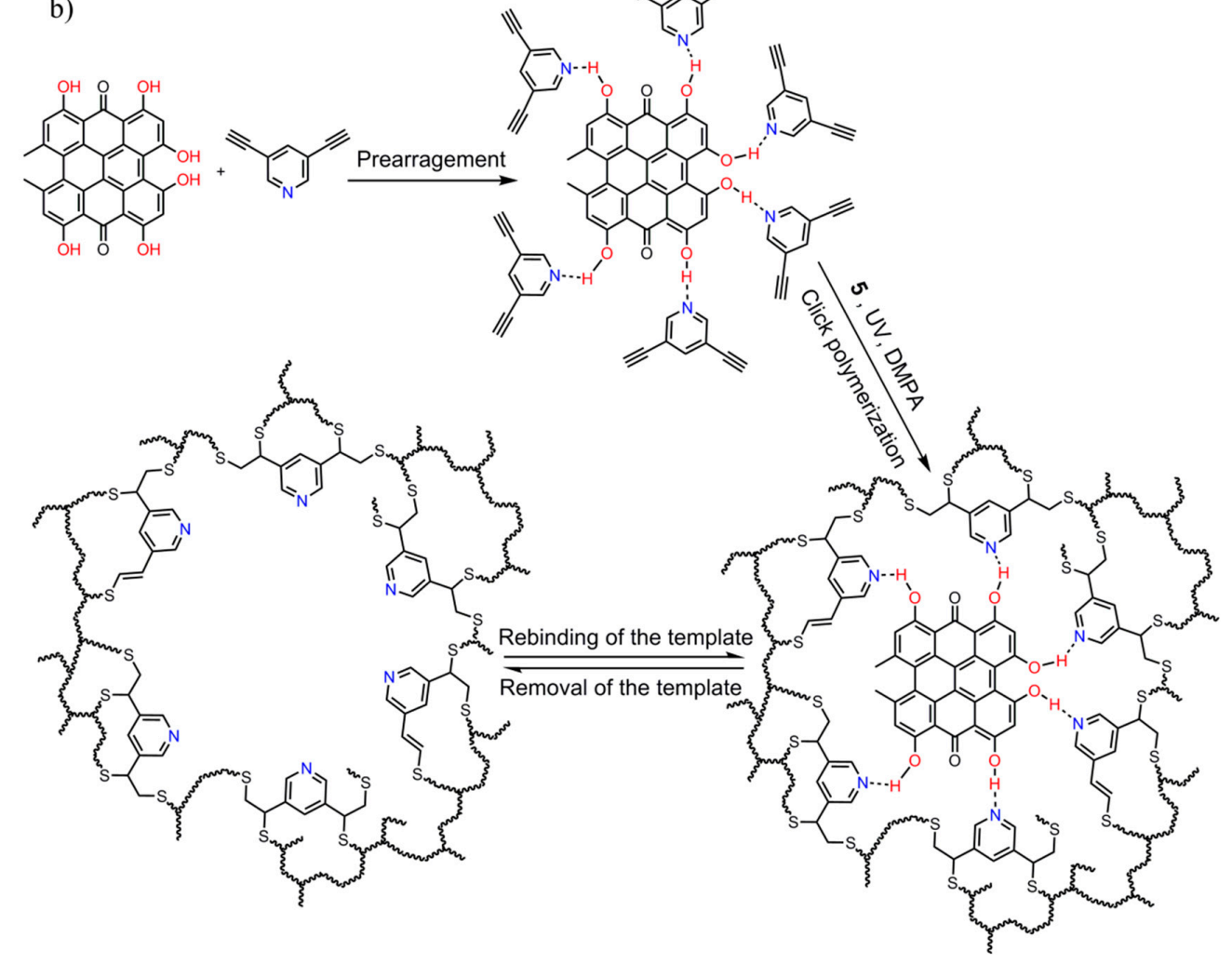

Scheme 1. (a) Illustration of hydrogen bonding of template, hypericin with functional monomer 1; (b) Schematic illustration of fabricating MIP-NSHs with $\mathbf{1}$ as monomer and $\mathbf{5}$ as crosslinker.

\section{Experimental Section}

\subsection{Chemicals and Instrumentation}

The chemicals 3,5-dibromopyridine (98\%), 2,6-dibromopyridine (98\%) were purchased from Daejeon Fung Teng Co., Ltd. (Beijing, China). The chemical 2-methyl-3-butyn-2-ol (98\%) was purchased from J \& K (Beijing, China). Copper(I) Iodide (98\%), trimethylolpropane (>98\%), pentaerythritol (98\%), bis(triphenylphosphine)palladium(II) chloride (Pd 15.2\%), and sodium methylate (97\%) were purchased from Aladdin (Shanghai, China). Trimethylsilylacetylene (98\%) was purchased from Energy Chemical (Shanghai, China). Benzoin dimethyl ether (DMPA) (98\%) was purchased from Heowns (Tianjin, China). Sodium azide (99\%) was purchased from Xiya Reagent Co., Ltd. (Chengdu, China). Potassium hydroxide, acetonitrile, acetone, DMSO were purchased from Kelon Chemical Co., Ltd. (Chengdu, China). Toluene, triethylamine, methanol, sulfuric acid (98\%), and all the other reagents were purchased from Tianjin Bodi Chemical Industry Co, Ltd. (Tianjin, China). Emodin was purchased from Tianfeng Biological Technology Co, Ltd. (Xi'an, China). Protohypericin (Protohyp) and hypericin 
(Hyp) were synthesized according to the procedures developed in our lab [33], and characterized by ${ }^{1} \mathrm{H}$ NMR (see Supplementary Materials Figures S14 and S15, respectively).

NMR spectra were recorded on a Bruker $500 \mathrm{MHz}$ Spectrometer (Bruker, Fällanden, Switzerland) with working frequencies of $500 \mathrm{MHz}$ for ${ }^{1} \mathrm{H}$ in DMSO- $d_{6}, \mathrm{CDCl}_{3}$, or MeOH- $d_{4}$. The residual signals from DMSO- $d_{6}\left({ }^{1} \mathrm{H}: \delta 2.50 \mathrm{ppm}\right), \mathrm{CDCl}_{3}\left({ }^{1} \mathrm{H}: \delta 7.26 \mathrm{ppm}\right)$, or $\mathrm{MeOH}-d_{4}\left({ }^{1} \mathrm{H}: \delta 3.31 \mathrm{ppm}\right)$ were used as internal standards. Dynamic light scattering (DLS) analysis was performed on a DelsaTM Nano system (Beckman Coulter, Brea, CA, USA). The surface area and the porosity of the prepared polymeric nanospheres were determined using nitrogen physisorption based on the Brunauer-Emmet-Teller (BET) method (ASAP 2020 HD88, Micromeritics Inc., Norcross, GA, USA). Samples were vacuum-degassed at $50{ }^{\circ} \mathrm{C}$ for $9 \mathrm{~h}$ before the adsorption experiments. Field emission scanning electron microscopy (FESEM) images were obtained with a Hitachi-S4800 instrument (Hitachi, Tokyo, Japan) operating at $10 \mathrm{kV}$. High performance liquid chromatography (HPLC) was performed with C18 reversed-phase column $\left(5 \mu \mathrm{m}, 4.6 \mathrm{~mm} \times 150 \mathrm{~mm}\right.$, Inertsil ODS-SP, Shimadzu, Tokyo, Japan) at $25^{\circ} \mathrm{C}$. The mobile phase consisted of $50 \%$ acetonitrile, $50 \%$ of the mixture of ammonium acetate-acetic acid buffer $(0.3 \mathrm{M}$, $\mathrm{pH}=6.96)$, and methanol $(1: 4, v / v)$; detection wavelength: $590 \mathrm{~nm}$; flow rate: $0.4 \mathrm{~mL} / \mathrm{min}$; injection volume: $10 \mu \mathrm{L}$. The retention time of hypericin under the given chromatographic system is $15.21 \mathrm{~min}$.

\subsection{Synthesis of Monomers and Crosslinkers}

As phenol hydroxy groups in hypericin are slightly acidic, we chose 3,5-diethynyl-pyridine (1) and 2,6-diethynyl-pyridine (2) as the monomers. Three crosslinkers of 1,3-bis(azidoacetoxy)-2-azidoacetoxymethyl-2-ethylpropane (tri-azide, 3), 2,2-bis((2-azidoacetoxy)methyl)propane-1,3-diyl bis(2-azidoacetate) (tetra-azide, 4), and trimethylolpropane tris(3-mercaptopropionate) (tri-thiol, 5) were chosen for azide-alkyne or thiol-yne click reactions in this work (see Figure 1). 1-5 were synthesized by following the published procedures [30,34-37] and characterized by ${ }^{1} \mathrm{H}$ NMR. The synthetic details can be found in Supplementary Materials and their ${ }^{1} \mathrm{H}$ NMR spectra were shown in Figure S1-S5, S9-S13).

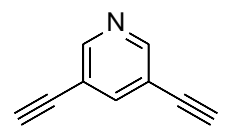

1

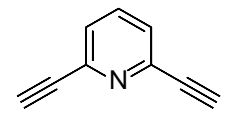

2<smiles>CCC(COC(=O)CN)(COC(=O)CN)COC(=O)CN</smiles>

3<smiles>[NH]CC(=O)OCC(COC(=O)CN)(COC(=O)CN)COC(=O)CN</smiles>

4

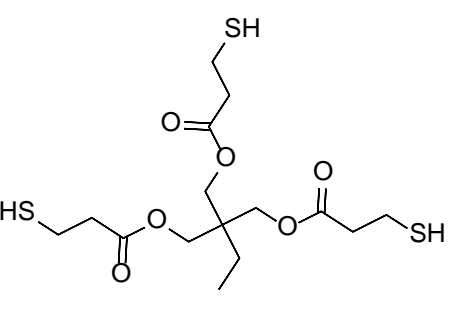

5

Figure 1. The chemical structures of monomers (1-2) and crosslinkers (3-5) used for synthesis of MIP-NSHs.

\subsection{Preparation of Imprinted Polymer Nanospheres towards Hypericin and Non-Imprinted Polymer Nanospheres}

Typically, polymer nanospheres imprinted towards hypericin (MIP-NSHs) were prepared as follows. To a quartz tube, $0.1 \mathrm{mmol}$ functional monomer, $0.1 \mathrm{mmol}$ cross-linker, $0.031 \mathrm{mmol}$ DMPA, $2 \mathrm{~mL}$ acetone were added, and the mixture was subjected to ultrasonication for $10 \mathrm{~min}$, followed by adding $5 \mu \mathrm{mol}$ template. The click polymerization was performed for $4 \mathrm{~h}$ under UV light at a wavelength of $350 \mathrm{~nm}$ at ambient temperature under $\mathrm{N}_{2}$ and stirring. After the reaction, the resulting 
beads were collected, filtered, and extracted to remove hypericin. Then the polymer beads were allowed to dry naturally and grounded with a pestle for 15 min to obtain MIP-NSHs.

The extraction procedure: The nanospheres were extracted with a soxhlet extractor using $10 \%$ acetic acid in acetone for $48 \mathrm{~h}$, thereafter the nanoshperes were ultrasonicated in $5 \mathrm{~mL} \mathrm{20 \%}$ acetic acid in acetone for $20 \mathrm{~min}$ and then centrifuged; the resulting supernatant was monitiored using HPLC. The ultrasonication step was repeated until no hypericin could be detected in the supernatant.

Control polymer nanospheres (NIP-NSs) were prepared under identical conditions but in the absence of hypericin.

\subsection{Determination of Static Adsorption Capacity}

To determine the static adsorption capacity, $5 \mathrm{mg}$ of the nanospheres (MIP-NSHs or NIP-NSs) was placed into a $10 \mathrm{~mL}$ plastic centrifuge tube and mixed with $5 \mathrm{~mL}$ of the test molecule acetone solution $(10.0 \mu \mathrm{M})$ to allow adsorption at $25^{\circ} \mathrm{C}$ for $24 \mathrm{~h}$. The concentration of the test molecule in the supernatant was measured with HPLC using a calibration curve (see Supplementary Materials Figure S6). The adsorption capacity $\left(Q, \mu \mathrm{mol} \cdot \mathrm{g}^{-1}\right)$ was calculated according to Equation 1 :

$$
Q=\frac{\left(C_{0}-C_{\mathrm{e}}\right) V}{W}
$$

where $C_{0}$ and $C_{\mathrm{e}}$ represent the initial and equilibrium concentrations of the test molecule in acetone $(\mu \mathrm{M})$, respectively, $V(\mathrm{~L})$ is the volume of the solution, and $W(\mathrm{~g})$ is the dry weight of the nanospheres.

The neat adsorption capacity of MIP-NSHs over that of NIP-NSs, defined as the specific adsorption capacity of MIP-NSHs $\left(Q_{s}\right)$ towards hypericin, is calculated according to Equation 2:

$$
Q_{\mathrm{s}}=Q_{1}-Q_{2}
$$

where $Q_{1}$ and $Q_{2}$ are the static adsorption capacity of MIP-NSHs and NIP-NSs $\left(\mu \mathrm{mol} \cdot \mathrm{g}^{-1}\right)$ towards hypericin, respectively.

\subsection{Kinetic of Template Adsorption}

$5.0 \mathrm{mg}$ of MIP-NSHs (or NIP-NSs) were mixed with a hypericin acetone solution $(12.5 \mu \mathrm{M}, 5 \mathrm{~mL}$ ) in a $10-\mathrm{mL}$ centrifuge tube. The tube was sealed and shaken in the dark at $25^{\circ} \mathrm{C}$ for different time intervals $(0.5,1,2,4,8$, and $12 \mathrm{~h}$, respectively). The concentrations of hypericin in the supernatant were determined by HPLC. The respective adsorptions were then calculated according to Equation 1.

\subsection{Isotherm Adsorption}

Six portions of $5.0 \mathrm{mg}$ polymers were weighed into plastic centrifuge tubes and mixed with $5 \mathrm{~mL}$ of hypericin acetone solution with different concentrations $(2.5,5.0,10.0,12.5,25$, and $50.0 \mu \mathrm{M})$, respectively. The mixtures were shaken for $8 \mathrm{~h}$ at $25^{\circ} \mathrm{C}$, and the concentrations of hypericin in the supernatants were determined by HPLC. The respective adsorptions were then calculated according to Equation 1. The isotherm adsorption curve was plotted based on the concentrations of hypericin in supernatants versus the amount of hypericin bound to MIP-NSHs.

\subsection{Selectivity of MIP-NSHs and NIP-NSs for Hypericin}

The binding selectivity of MIP-NSHs and NIP-NSs was evaluated by following the method reported [38]. Briefly, $5 \mathrm{mg}$ of the MIP-NSHs or NIP-NSs were incubated respectively with $5 \mathrm{~mL}$ of hypericin, protohypericin, and emodin solution $\left(12.5 \mu \mathrm{M}\right.$ in acetone) at $25^{\circ} \mathrm{C}$. After incubation under continuous shaking for $24 \mathrm{~h}$, the amounts of hypericin, protohypericin, and emodin bound to the MIP-NSHs or NIP-NSs were measured, respectively. The binding selectivity of the NSs towards 
different molecules was compared using the "selectivity factor" (SF) and "imprinting factor" (IF) [39], which are determined as the ratio of distributions and can be calculated by the following equations:

$$
\begin{gathered}
D=\frac{C_{0}-C_{\mathrm{e}}}{C_{\mathrm{e}}} \\
S F=\frac{\mathrm{D}_{\mathrm{MIP}}^{\mathrm{h}}}{D_{\mathrm{MIP}}^{\prime}}=\frac{\frac{\left(C_{0, \mathrm{MIP}}^{\mathrm{h}}-C_{\mathrm{e}, \mathrm{MIP}}^{\mathrm{h}}\right)}{C_{\mathrm{e}, \mathrm{MIP}}^{\mathrm{h}}}}{\frac{\left(\mathrm{C}_{0, \mathrm{MIP}}^{\prime}-\mathrm{C}_{\mathrm{e}, \mathrm{MIP}}^{\prime}\right)}{C_{\mathrm{e}, \mathrm{MIP}}^{\prime}}}
\end{gathered}
$$

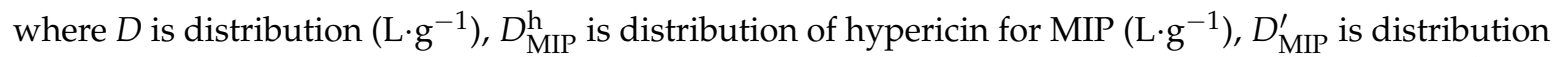
of competitor for MIP $\left(\mathrm{L} \cdot \mathrm{g}^{-1}\right), C_{0, \mathrm{MIP}}^{\mathrm{h}}$ is the initial concentration of hypericin for MIP $(\mu \mathrm{M}), C_{\mathrm{e}, \mathrm{MIP}}^{\mathrm{h}}$ is the equilibrium concentration of hypericin for MIP $(\mu \mathrm{M}), C_{0, \text { MIP }}^{\prime}$ is the initial concentration of competitor for MIP $(\mu \mathrm{M})$, and $C_{e, \text { MIP }}^{\prime}$ is the equilibrium concentration of competitor for MIP $(\mu \mathrm{M})$.

$$
I F=\frac{D_{\mathrm{MIP}}}{D_{\mathrm{NIP}}}=\frac{\frac{\left(C_{0, \mathrm{MIP}}-C_{\mathrm{e}, \mathrm{MIP}}\right)}{C_{\mathrm{e}} \mathrm{MIP}}}{\frac{\left(C_{0, \mathrm{NI}}-C_{\mathrm{e}, \mathrm{NIP}}\right)}{C_{\mathrm{e}}, \mathrm{NIP}}}
$$

where $D_{\mathrm{MIP}}$ is the distribution of the test molecule for MIP $\left(\mathrm{L} \cdot \mathrm{g}^{-1}\right), D_{\mathrm{NIP}}$ is the distribution of the test

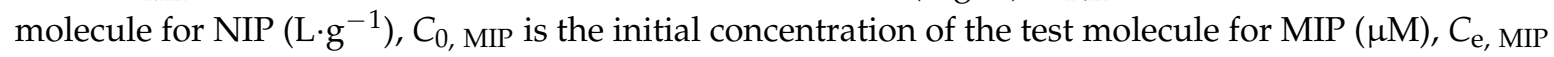
is the equilibrium concentration of the test molecule for MIP $(\mu \mathrm{M}), C_{0}$, NIP is the initial concentration of the test molecule for NIP $(\mu \mathrm{M})$, and $C_{e}$, NIP is the initial concentration of the test molecule for NIP $(\mu \mathrm{M})$.

\subsection{The Reusability of MIP-NSHs}

The reusability of MIP-NSHs was evaluated by an adsorption-extraction cycle experiment. One adsorption-extraction cycle consisted of loading the template and reaching equilibrium adsorption, followed by the extraction of the template. For the adsorption, $5 \mathrm{mg}$ of the MIP-NSHs were incubated with $5 \mathrm{~mL}$ of hypericin solution $\left(12.5 \mu \mathrm{M}\right.$ in acetone) at $25^{\circ} \mathrm{C}$. After incubation under continuous shaking for $8 \mathrm{~h}$, the concentration of hypericin in the supernatant was measured by HPLC. The adsorption capacity was calculated according to Equation 1. The MIP-NSHs were subjected to the extraction procedure mentioned above, and then used for the next cycle.

\section{Results and Discussion}

\subsection{Synthesis of MIP-NSHs}

\subsubsection{Screening of Monomers and Crosslinkers}

Previously, we have successfully prepared polymeric nanospheres via the alkyne-azide cycloaddition between either of the monomers and either of the cross-linkers, i.e., four "monomer + crosslinker" combinations $(\mathbf{1}+3$ or $\mathbf{4}$ and $\mathbf{2}+\mathbf{3}$ or $\mathbf{4})$ initiated by $\mathrm{Cu}\left(\mathrm{PPh}_{3}\right)_{3} \mathrm{Br}$ [30]. However, due to the low polymer yield from the initiation system, a new photoinitiatior of DMPA was used for polymerization in this work. The four combinations were used for the preparation of imprinted polymers (denoted as P1, P2, P3, and P4 correspondingly) towards hypericin. The adsorption study showed that the MIP-NSHs obtained from $\mathbf{1}+\mathbf{3}$ (Table 1, P1) gave the highest specific adsorption capacity $\left(Q_{\mathrm{s}}=1.91 \mu \mathrm{mol} \cdot \mathrm{g}^{-1}\right)$ among the four polymers, as shown in Table 1 . Under the same conditions, $Q_{s}$ from $2+3$ (Table $1, \mathrm{P} 2$ ) was far lower at $0.33 \mu \mathrm{mol} \cdot \mathrm{g}^{-1}$. The reason may be that the steric hindrance of $\mathbf{2}$ is greater than that of $\mathbf{1}$ when forming the hydrogen bond between the $\mathrm{N}$ atom in pyridine ring and the phenolic hydroxyl groups in hypericin, which consequently affects the creation of the imprinting sites and thus impacts the recognition towards hypericin. 
Table 1. The specific adsorption capacity $\left(Q_{s}\right)$ of the MIP-NSHs towards hypericin.

\begin{tabular}{cccc}
\hline Polymer & Combination & $Q_{s}\left(\mu \mathrm{mol} \cdot \mathrm{g}^{-\mathbf{1}}\right)$ & $\operatorname{RSD}(\boldsymbol{n}=\mathbf{3})$ \\
\hline P1 & $\mathbf{1 + 3}$ & 1.911 & 0.022 \\
P2 & $\mathbf{2}+\mathbf{3}$ & 0.331 & 0.070 \\
P3 & $\mathbf{1}+\mathbf{4}$ & 0.452 & 0.069 \\
P4 & $\mathbf{2}+\mathbf{4}$ & 0.745 & 0.038 \\
P5 & $\mathbf{1} \mathbf{5}$ & 2.204 & 0.032 \\
\hline
\end{tabular}

In addition, using tetra-azide 4 instead of tri-azide 3 as the crosslinker did not benefit the adsorption of the resulting MIP-NSHs. For example, the $Q_{\mathrm{s}}$ of P3 synthesized from $1+4$ was much lower than that of P1. This might be because the polymer formed with 4 as crosslinker was denser than that with 3 as crosslinker, which make the removal and re-binding of hypericin from the polymer more difficult, and, as a result, lowered the adsorption of MIP-NSHs drastically. Thus, a tri-thiol crosslinker 5 was synthesized and combined with 1 to fabricate MIP-NSHs via thiol-yne click reaction (Table 1, P5). As expected, the $Q_{\mathrm{s}}$ obtained was the best among the five tested combinations, which may be due to the absence of triazole rings and less rigid structure of P5. Therefore, the subsequent studies were based on the MIP-NSHs obtained from combination of monomer $\mathbf{1}$ and crosslinker $\mathbf{5}$.

\subsubsection{Characterization of MIP-NSHs}

\section{FTIR Analysis}

To confirm that the thiol-yne click polymerization took place between $\mathbf{1}+\mathbf{5}$ in the presence or absence of hypericin, both MIP-NSHs and NIP-NSs were analyzed using FTIR spectroscopy. The IR spectra are shown in Figure 2. In both scenarios, the characteristic absorption of $C=O$ from 5 at $1735 \mathrm{~cm}^{-1}$, 2103,3274 , and $2570 \mathrm{~cm}^{-1}$ ascribed to the absorptions of $\mathrm{C} \equiv \mathrm{C}, \mathrm{C}-\mathrm{H}$ in alkynyl groups on $\mathbf{1}$ [30] and S-H on 5, respectively were not observed on the spectra of the polymers (MIP-NSHs and NIP-NSs). Moreover, the absorption band of $\mathrm{C}-\mathrm{O}$ on hypericin at $1230 \mathrm{~cm}^{-1}$ [38] was found on the spectrum of MIP-NSHs. The results indicated that the click reaction of thiol-yne successfully occurred in both cases.

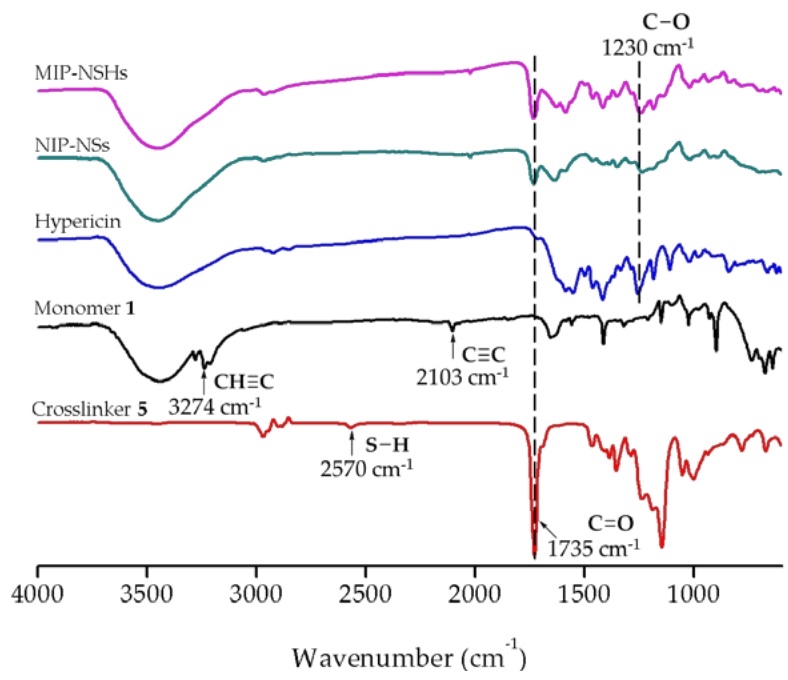

Figure 2. FTIR spectra of MIP-NSHs, NIP-NSs, Monomer 1, Crosslinker 5 and Hypericin.

\section{SEM and DLS Analysis}

The polymers obtained via click polymerization between $\mathbf{1}+\mathbf{5}$ were characterized with FESEM and DLS analyses. The results are shown in Figure 3, Table 2, and Figure S7 (see Supplementary Materials). As shown in Figure 3, both of the imprinted and non-imprinted polymeric nanoparticles 
showed good spherical morphology. The difference between MIP-NSHs and NIP-NSs was that the surface of the former is much rougher, which can be clearly seen in the enlarged views (insets in Figure 3). DLS analysis showed that the average diameters of the MIP-NSHs and NIP-NSs were 677 and $497 \mathrm{~nm}$, respectively; their polydispersity indexes were 1.137 and 0.994 , respectively. In addition, the extraction process yielded an obvious change in the $\zeta$ potential of the MIP-NSHs, from 0.95 to $-13.51 \mathrm{mV}$; in contrast, the $\zeta$ potential of NIP-NSs was less affected (see Table 2). This is in accordance with what has been reported previously in the literature [38]. The difference in the $\zeta$ potentials of MIP-NSHs before and after the extraction process is most likely due to the removal of hypericin molecules from the MIP-NSHs. All these results confirm that the MIP-NSHs can be prepared via thiol-yne click reaction of $\mathbf{1}+\mathbf{5}$.

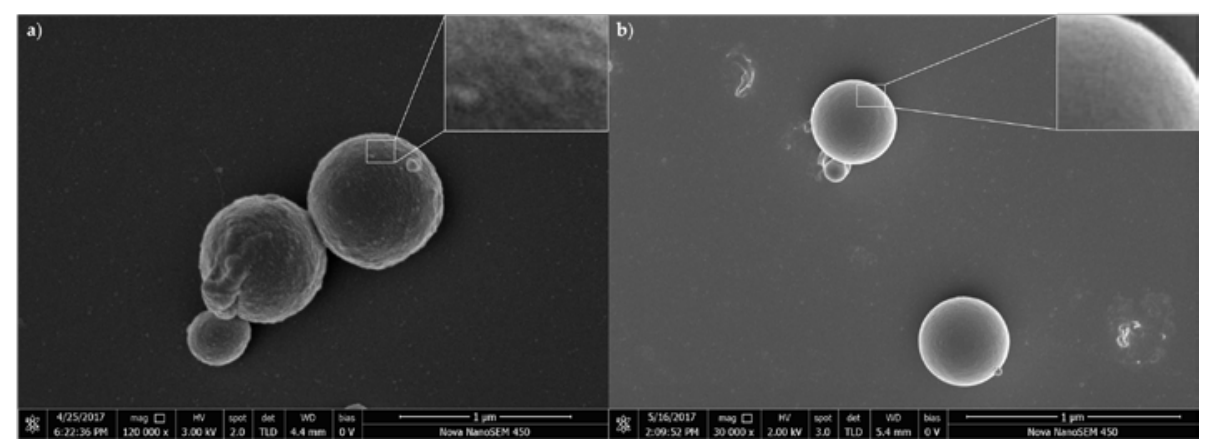

Figure 3. SEM images of the nanospheres from $1+5$ : (a) MIP-NSHs; (b) NIP-NSs. The insets are enlarged views correspondingly. Scale bar: $1 \mu \mathrm{m}$.

Table 2. The DLS data of the NSs prepared.

\begin{tabular}{cccc}
\hline NPs & Paticle Size $(\mathbf{n m})$ & Polydispersity Index & $\zeta$ Potential $(\mathbf{m V})$ \\
\hline MIP-NSHs & $677 \pm 68$ & 1.137 & $0.95 \pm 0.90$ \\
NIP-NSs & $497 \pm 80$ & 0.994 & $-2.86 \pm 0.52$ \\
MIP-NSHs $^{1}$ & $661 \pm 98$ & 0.609 & $-13.51 \pm 0.17$ \\
NIP-NSs $^{1}$ & $464 \pm 75$ & 0.874 & $-1.42 \pm 0.56$ \\
\hline \multicolumn{4}{c}{}
\end{tabular}

\section{BET Analysis}

The average pore diameter, surface area and pore volume of the MIP-NSHs and NIP-NSs fabricated in this work were characterized with BET analysis. The results are summarized in Table 3 and Figure S8 (see Supplementary Materials). As can be seen, the average pore diameter and the pore volume of the MIP-NSHs were $52.839 \mathrm{~nm}$ and $0.033 \mathrm{~cm}^{3} \cdot \mathrm{g}^{-1}$, respectively; which are much larger than those of NIP-NSs, indicating that the former has a porous structure, and the latter a compact one. The average pore diameter, surface area and pore volume of the MIP-NSHs were roughly 13, 2.5 and 8.4 times those of the NIP-NSs, respectively.

Table 3. The pore size, the surface area, and the pore volume of the NSs.

\begin{tabular}{|c|c|c|c|}
\hline NPs & $\begin{array}{c}\text { Average Pore } \\
\text { Diameter (nm) }\end{array}$ & $\begin{array}{l}\text { Surface Area } \\
\left(\mathrm{m}^{2} \cdot \mathrm{g}^{-1}\right)\end{array}$ & $\begin{array}{l}\text { Pore Volume } \\
\left(\mathrm{cm}^{3} \cdot \mathrm{g}^{-1}\right)\end{array}$ \\
\hline MIP-NSHs & $52.839 \pm 0.393$ & $5.274 \pm 0.517$ & $0.0330 \pm 0.0299$ \\
\hline NIP-NPs & $4.139 \pm 0.0522$ & $2.083 \pm 0.266$ & $0.00392 \pm 0.0726$ \\
\hline
\end{tabular}




\subsubsection{Optimization of Preparation Conditions for Specific Adsorption Capacity of MIP-NSHs}

In order to obtain MIP-NSHs with the most optimal imprinting effect towards hypericin, the preparation conditions that affected $Q_{\mathrm{s}}$ were screened and optimized; this included photoinitiator concentration, solvent composition, hypericin concentration, the equivalent ratio of $\mathbf{1}$ to 5 , etc.

Effect of Photoinitiator Concentration on $Q_{\mathrm{s}}$

As previously mentioned, DMPA was used as the photoinitiator in this work, which generated active free radicals upon exposure to UV light and induced monomer and crosslinker click polymerization. The concentration of DMPA affected not only the rate of polymerization, but also the imprinting effect of the resulting imprinted polymers. The effect of the concentration of DMPA on $Q_{\mathrm{s}}$ was investigated by varying the concentration of DMPA in a range of $2.0-16 \mathrm{mg} \cdot \mathrm{mL}^{-1}$. As shown in Table 4 , when the concentration of DMPA increased from 2.0 to $4.0 \mathrm{mg} \cdot \mathrm{mL}^{-1}, Q_{\mathrm{s}}$ increased dramatically from 0.65 to $2.71 \mu \mathrm{mol} \cdot \mathrm{g}^{-1}$. However, further increase in the concentration led to the opposite effect with $Q_{\mathrm{s}}$ decreasing to $0.57 \mu \mathrm{mol} \cdot \mathrm{g}^{-1}$ when the concentration was increased to $16 \mathrm{mg} \cdot \mathrm{mL}^{-1}$. Hence, $4 \mathrm{mg} \cdot \mathrm{mL}^{-1}$ was used as the optimal concentration of the photoinitiator for subsequent studies.

Table 4. The effect of photoinitiator concentration on the specific adsorption capacity.

\begin{tabular}{cccc}
\hline Polymer & Concentration of DMPA $\left(\mathbf{m g} \cdot \mathbf{m L}^{-\mathbf{1}}\right)$ & $Q_{s}\left(\mu \mathbf{m o l} \cdot \mathbf{g}^{-\mathbf{1}}\right)$ & RSD $(\boldsymbol{n}=\mathbf{3})$ \\
\hline P6 & 2.0 & 0.64 & 0.035 \\
P7 & 4.0 & 2.16 & 0.095 \\
P8 & 8.0 & 1.09 & 0.046 \\
P9 & 16.0 & 0.57 & 0.089 \\
\hline
\end{tabular}

Effect of Solvent Composition on $Q_{\mathrm{s}}$

Solvent plays an important role in the process of preparing MIP, especially in a non-covalent imprinting process. Proper selection of solvent may promote the formation of non-covalent adducts between functional monomers and templates and enhance the efficiency of imprinting [40]. Considering the solubility of hypericin, acetone was used in this study as a component of the solvent medium for polymerization. The effect of the ratio of acetone to acetonitrile on $Q_{\mathrm{s}}$ of MIP-NSHs was studied by varying the ratio in a range of 4:0 to $1: 3(v / v)$. The results showed that the best ratio of acetone to acetonitrile was 3:1, where $Q_{\mathrm{s}}$ reached $2.24 \mu \mathrm{mol} \cdot \mathrm{g}^{-1}$ (Table 5); lower ratios led to a dramatic decrease in $Q_{\mathrm{s}}, 1.47 \mu \mathrm{mol} \cdot \mathrm{g}^{-1}$ with $1: 1$, and $0.29 \mu \mathrm{mol} \cdot \mathrm{g}^{-1}$ with $1: 3$.

Table 5. The effect of solvent composition on $Q_{s}$.

\begin{tabular}{cccc}
\hline Polymer & Acetone/Acetonitrile $(v / v)$ & $Q_{s}\left(\mu \mathrm{mol} \cdot \mathbf{g}^{-\mathbf{1}}\right)$ & RSD $(\boldsymbol{n}=\mathbf{3})$ \\
\hline P7 & $4: 0$ & 2.16 & 0.095 \\
P10 & $3: 1$ & 2.23 & 0.036 \\
P11 & $1: 1$ & 1.47 & 0.048 \\
P12 & $1: 3$ & 0.28 & 0.079 \\
\hline
\end{tabular}

Effect of Template Concentration and the Ratio of Monomer to Crosslinker on $Q_{\mathrm{s}}$

The template concentration and ratio of monomer to crosslinker have a crucial influence on the property of MIPs [41]. They are important factors in dictating the number of binding sites and the rigidity of MIPs [42]. When there is sufficient amount of monomers for the pre-assembly of template and monomer, an increasing amount of template ensures a bigger number of binding sites and therefore a higher adsorption capacity, while an excessive crosslinker concentration (or too low ratio of monomer to crosslinker) leads to a highly crosslinked polymer, which impedes the template to transport in and out of the binding sites and thus decreases the adsorption capacity. This theory was further confirmed in this work. As shown in Table 6, when the concentration of hypericin increased from 0.625 to $2.5 \mathrm{mg} \cdot \mathrm{mL}^{-1}$, 
$Q_{\mathrm{s}}$ increased from 1.35 to $2.57 \mu \mathrm{mol} \cdot \mathrm{g}^{-1}$, however, further increase in the concentration of hypericin to $5.0 \mathrm{mg} \cdot \mathrm{mL}^{-1}$ resulted in a clear decrease of $Q_{\mathrm{s}}$ (see Table $6 \mathrm{P} 15$ ). As the equivalent ratio of 1 to 5 decreased from $4: 1$ to $1: 2, Q_{\mathrm{s}}$ increased at first, from 0.59 to a maximum value of $2.57 \mu \mathrm{mol} \cdot \mathrm{g}^{-1}$, and subsequently decrease dramatically to $0.45 \mu \mathrm{mol} \cdot \mathrm{g}^{-1}$ when the ratio decreased to 1:2.

Table 6. The effect of template molecule concentration on the specific adsorption capacity. ${ }^{1}$

\begin{tabular}{|c|c|c|c|c|}
\hline Polymer & $\begin{array}{l}\text { Template concentration } \\
\left(\mathbf{m g} \cdot \mathrm{mL}^{-1}\right)\end{array}$ & $\begin{array}{l}\text { Ratio of } 1 \text { to } 5 \\
\quad \text { (in equiv.) }\end{array}$ & $\underset{\left(\mu \mathrm{mol} \cdot \mathrm{g}^{-1}\right)}{Q_{s}}$ & $\begin{array}{c}\text { RSD } \\
(n=3)\end{array}$ \\
\hline P13 & 0.625 & $4: 3$ & 1.35 & 0.059 \\
\hline P7 & 1.25 & $4: 3$ & 2.17 & 0.095 \\
\hline P14 & 2.5 & $4: 3$ & 2.57 & 0.052 \\
\hline P15 & 5.0 & $4: 3$ & 1.52 & 0.039 \\
\hline P16 & 2.5 & $4: 1$ & 0.59 & 0.069 \\
\hline P17 & 2.5 & $3: 1$ & 1.10 & 0.093 \\
\hline P18 & 2.5 & 2:1 & 2.23 & 0.058 \\
\hline P19 & 2.5 & 1:1 & 1.94 & 0.049 \\
\hline P20 & 2.5 & $1: 2$ & 0.45 & 0.084 \\
\hline
\end{tabular}

Based on the results above, we conclude that the best combination of monomer/crosslinker for fabrication of MIP-NSHs in this work was $1+5$. The MIP-NSHs with an optimal $Q_{\mathrm{s}}$ value $\left(2.57 \mu \mathrm{mol} \cdot \mathrm{g}^{-1}\right)$ can be prepared under the following conditions: the concentratration of hypericin: $5.0 \mathrm{mM}$; the concentration of monomer $\mathbf{1}$ and crosslinker 5 is $50 \mathrm{mM}$ each; DMPA: $15.5 \mathrm{mM}$; solvent: acetone/acetonitrile in 3:1 $(v / v)$, room temperature, UV light at $350 \mathrm{~nm}, 4 \mathrm{~h}$. The MIP-NSH prepared under the optimal conditions described above, i.e. P14, were used for subsequent studies.

\subsection{Kinetic of Template Adsorption}

The equilibrium adsorption isotherms of MIP-NSHs and NIP-NSs for the binding of hypericin were investigated by batch adsorption experiments. As observed in Figure 4, the adsorption process of the MIP-NSHs displayed two phases: in the first phase, the adsorption amount increased quickly and reached to about one third of the total adsorption capacity during the first hour; and in the second phase, adsorption rate slowed down, and the equilibrium was reached at $8 \mathrm{~h}$. The first phase can be attributed to the binding of hypericin to the recognition sites located on the surface of MIP-NSHs, which bound hypericin molecules at a fast rate. While the second phase can be attributed to the binding of hypericin to the internal binding sites of MIPNSHs, where the diffusion of hypericin from the surface to the inner part of the nanospheres resulted in the slow adsorption rate once the surface recognition sites became saturated. In addition, the equilibrium adsorption capacity of MIP-NSHs is higher than that of NIP-NSs, which indicates a fair imprinting effect. 


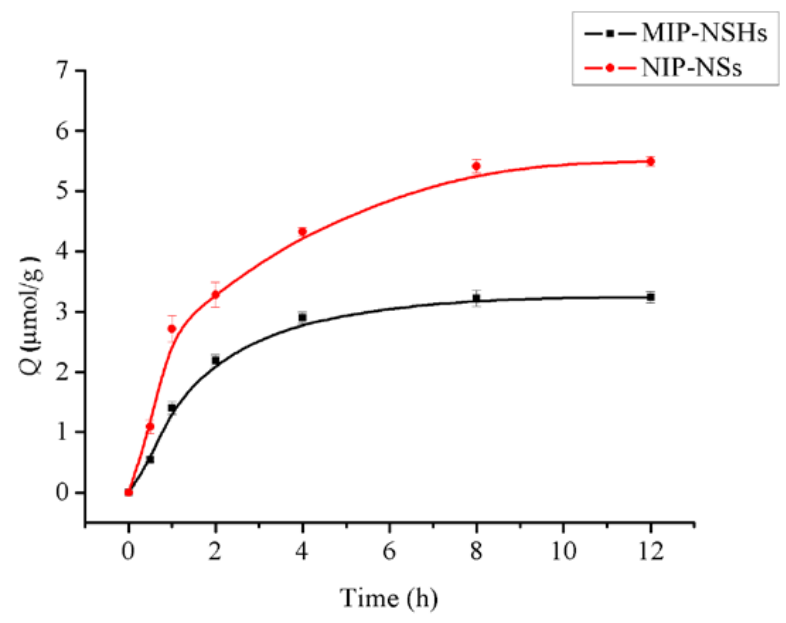

Figure 4. Dynamic adsorption curves of MIP-NSHs and NIP-NSs prepared with the optimal conditions.

\subsection{Affinity Analysis}

The binding experiments were performed at different initial concentrations of hypericin, ranging from 0 to $50.0 \mu \mathrm{M}$, to compare the $Q$ of the MIP-NSHs against that of NIP-NSs. Figure 5a shows the binding isotherms for hypericin on the MIP-NSHs and NIP-NSs. It can be seen that the $Q$ of MIP-NSHs increased quickly in a linear relationship with the concentration of hypericin before $C_{s}$, a critical concentration of $11.5 \mu \mathrm{M}$. Thereafter, the increase of the $Q$ slowed down and finally reached to a plateau. Furthermore, the amounts of substrate bound to the MIP-NSHs were more than that to the NIP-NSs, which was ascribed to the imprinting effect [43].

The adsorption mechanism study shows that the adsorption of MIP-NSHs towards hypericin fitted well with an extended Langmuir isotherm model (expressed by Equation 5, Figure 5b) [43,44], where $R^{2}$ is 0.9995 , equilibrium constant $K_{\mathrm{d}}=0.1843 \mu \mathrm{M}$, $\mathrm{m}$ is 1.8233 , and $Q_{\max }$ is $6.07 \mu \mathrm{mol} \cdot \mathrm{g}^{-1}$. The results indicate the absorption may be described by monolayer sorption on a non-smooth surface.

$$
\frac{C_{\mathrm{e}}^{m}}{Q}=\frac{1}{K_{\mathrm{d}} \cdot Q_{\max }}+\frac{C_{\mathrm{e}}^{m}}{Q_{\max }}
$$

$K_{d}$ is the equilibrium constant $(\mu \mathrm{M}), C_{e}$ is the equilibrium concentration of hypericin $(\mu \mathrm{M})$ in supernatant, and $Q_{\max }$ is the apparent maximum absorption capacity of binding sites $\left(\mu \mathrm{mol} \cdot \mathrm{g}^{-1}\right)$.

a)

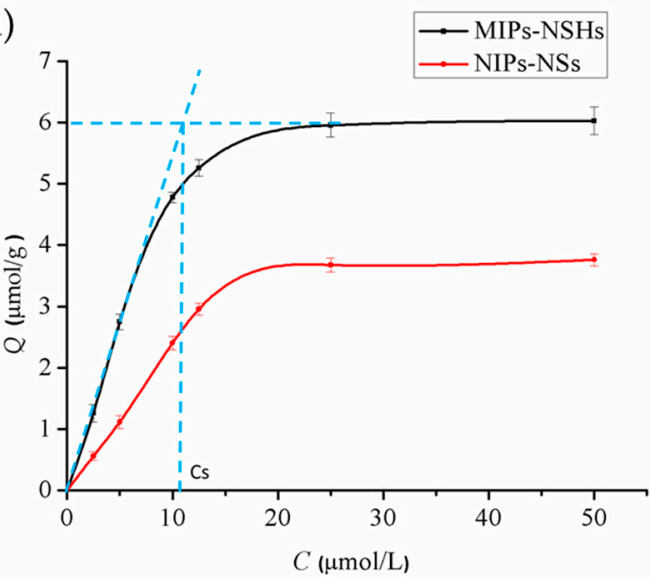

b)

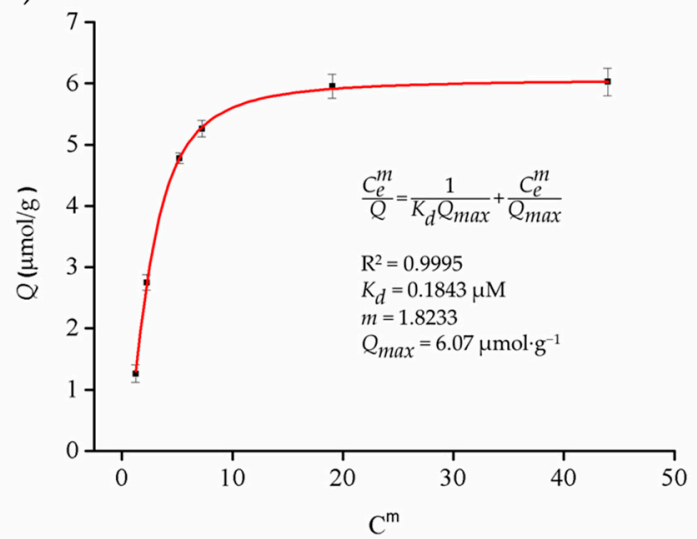

Figure 5. (a) Isothermal adsorption curve of MIP-NSHs and NIP-NSs; (b) The fitting curve of the extended Langmuir adsorption. 


\subsection{Binding Selectivity}

The binding selectivity of MIP-NSHs towards hypericin was examined by using a similar method reported previously [38], where protohypericin and emodin were used as competitors of the template (their structures are displayed in Figure 6a). MIP-NSHs or NIP-NSs were incubated respectively with the same amount of hypericin, protohypericin, and emodin under the same conditions. The respective adsorption capacities of MIP-NSHs and NIP-NSs towards the three molecules are shown in Figure $6 \mathrm{~b}$. A higher adsorption for hypericin $\left(6.03 \mu \mathrm{mol} \cdot \mathrm{g}^{-1}\right)$ was obtained with MIP-NSHs compared to protohypericin and emodin (2.36 and $1.30 \mu \mathrm{mol} \cdot \mathrm{g}^{-1}$, respectively). The binding selectivity of the NSs was evaluated with SF and IF, respectively. SF of MIP-NSHs towards protohypericin and emodin was 3.34 and 8.04, respectively; IF of MIP-NSHs towards hypericin, protohypericin, and emodin, was $2.44,2.88$, and 2.10 , respectively (Table 7 ).

a)

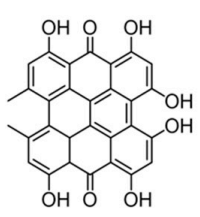

Hypericin

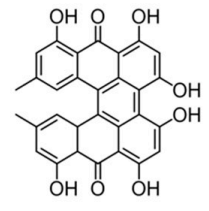

Protohypericin

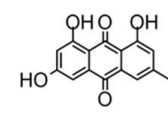

Emodin b)

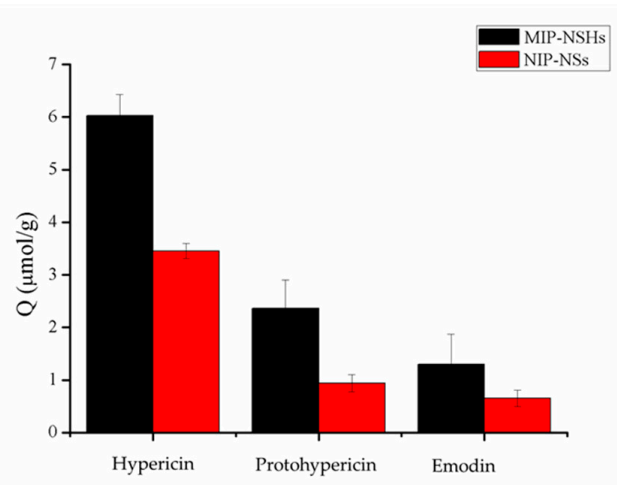

Figure 6. (a) The chemical structures of hypericin, protohypericin, and emodin; (b) Selective bindings of MIP-NSHs (black) and NIP-NSs (red) toward to hypericin, protohypericin, and emodin, respectively.

Table 7. The values of $S F$ and $I F$.

\begin{tabular}{cccc}
\hline Factor & Hypericin & Protohypericin & Emodin \\
\hline$S F$ & $/$ & 3.34 & 8.04 \\
$I F$ & 2.44 & 2.88 & 2.10 \\
\hline
\end{tabular}

\subsection{Reusability of MIP-NSHs}

Imprinting materials as chemosensors are robust materials that are supposed to be reused many times, which is essential for reliable, economical and sustainable applications [45]. The evaluation on the reusability of the MIP-NSHs was performed and the results are shown in Figure 7. It can be seen that after five adsorption-extraction cycles, the adsorption capacity of the MIP-NSHs remained high, indicating good stability. 


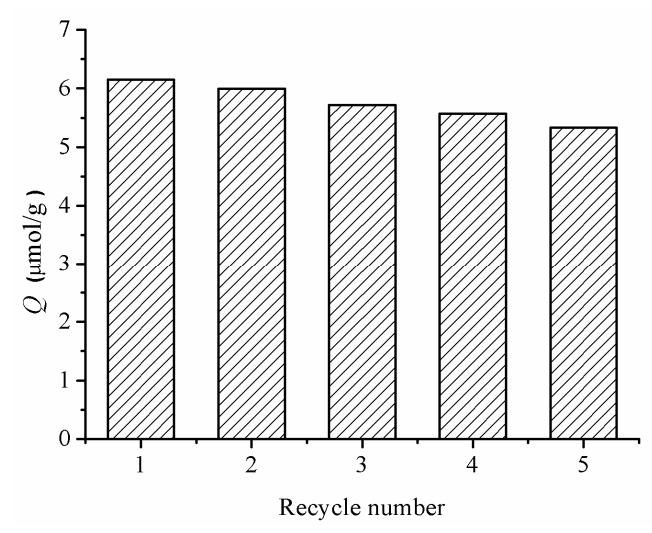

Figure 7. Reusability of MIP-NSHs.

\section{Conclusions}

In conclusion, MIP-NSHs were successfully synthesized via thiol-yne click reaction using 3,5-diethynyl-pyridine as the monomer, tris(3-mercaptopropionate) as the crosslinker, and hypericin as the template. The click polymerization was completed in merely $4 \mathrm{~h}$ to produce the desired MIP-NSHs. The reaction conditions for adsorption capacity and selectivity towards hypericin were optimized, and the MIP-NSHs synthesized under the optimized conditions showed a high adsorption capacity $\left(Q=6.03 \mu \mathrm{mol} \cdot \mathrm{g}^{-1}\right)$ and fair selectivity towards hypericin. In addition, MIP-NSHs displayed good reusability up to at least five cycles. This work presents a straightforward method to fabricate molecularly imprinted nanospheres via thiol-yne click reaction.

Supplementary Materials: The following are available online at www.mdpi.com/2073-4360/9/10/469/s1, Figure S1: The synthesis of monomer 1, Figure S2: The synthesis of monomer 2, Figure S3: The synthesis of crosslinker 3, Figure S4: The synthesis of crosslinker 4, Figure S5: The synthesis of crosslinker 5, Figure S6: (a) Standard curve of hypericin in acetone by HPLC. (b) Standard curve of protohypericin in acetone by HPLC. (c) Standard curve of emodin in acetone by HPLC. HPLC detection conditions: C18 reversed-phase column $(5 \mu \mathrm{m}$, $4.6 \mathrm{~mm} \times 250 \mathrm{~mm}$, Shimadzu, Japan). The mobile phase consisted of $50 \%$ acetonitrile, $50 \%$ of the mixture of ammonium acetate-acetic acid buffer $(0.3 \mathrm{M}, \mathrm{pH}=6.96)$ and methanol $(1: 4, v / v)$; detection wavelength: $590 \mathrm{~nm}$; flow rate: $0.4 \mathrm{~mL} / \mathrm{min}$; injection volume: $10 \mu \mathrm{L}$, Figure S7: DLS histograms of MIP-NSHs and NIP-NSs before and after extracting process, Figure S8: The nitrogen adsorption and desorption isotherms of MIP-NSHs and NIP-NSs, Figure S9: The ${ }^{1} \mathrm{H}$ NMR spectrum of monomer 1, Figure S10: The ${ }^{1} \mathrm{H}$ NMR spectrum of monomer 2, Figure S11: The ${ }^{1} \mathrm{H}$ NMR spectrum of crosslinker 3, Figure S12: The ${ }^{1} \mathrm{H}$ NMR spectrum of crosslinker 4, Figure S13: The ${ }^{1} \mathrm{H}$ NMR spectrum of crosslinker 5, Figure S14: The ${ }^{1} \mathrm{H}$ NMR spectrum of protohypericin, Figure S15: The ${ }^{1} \mathrm{H}$ NMR spectrum of hypericin.

Acknowledgments: This research work was supported by the Project of Science and Technology of Social Development in Shaanxi Province (2016SF-029) and the National Natural Science Foundation of China (21174113 and 21772157).

Author Contributions: Yuxin Pei directed the entire research work and drafted the manuscript. Fengfeng Fan performed the experiments, analyzed the data, and drafted the experimental part. Xinxin Wang, Weiwei Feng, and Yong Hou performed the synthesis of the monomers and crosslinkers and analyzed the data. Zhichao Pei assisted with discussion, data analysis, and the revision of the manuscript.

Conflicts of Interest: The authors declare no conflict of interest.

\section{References}

1. Wulff, G. The use of polymers with enzyme-analogous structures for the resolution of racemates. Angew. Chem. Int. Ed. 1972, 11, 341.

2. Andersson, L.; Sellergren, B.; Mosbach, K. Imprinting of amino acid derivatives in macroporous polymers. Tetrahedron Lett. 1984, 25, 5211-5214. [CrossRef]

3. Pardeshi, S.; Singh, S.K. Precipitation polymerization: A versatile tool for preparing molecularly imprinted polymer beads for chromatography applications. RSC Adv. 2016, 6, 23525-23536. [CrossRef] 
4. Sun, G.; Liu, Y.; Ahat, H.; Shen, A.; Liang, X.; Xue, X.; Luo, Y.; Yang, J.; Liu, Z.-S.; Aisa, H.A. "Two-dimensional" molecularly imprinted solid-phase extraction coupled with crystallization and high performance liquid chromatography for fast semi-preparative purification of tannins from pomegranate husk extract. J. Chromatogr. A 2017, 1505, 35-42. [CrossRef] [PubMed]

5. Walshe, M.; Howarth, J.; Kelly, M.T.; OKennedy, R.; Smyth, M.R. The preparation of a molecular imprinted polymer to 7-hydroxycoumarin and its use as a solid-phase extraction material. J. Pharm. Biomed. Anal. 1997, 16, 319-325. [CrossRef]

6. Li, P.; Wang, T.; Lei, F.; Peng, X.; Wang, H.; Qin, L.; Jiang, J. Preparation and evaluation of paclitaxel-imprinted polymers with a rosin-based crosslinker as the stationary phase in high-performance liquid chromatography. J. Chromatogr. A 2017, 1502, 30-37. [CrossRef] [PubMed]

7. Muldoon, M. Application of molecularly-imprinted polymers for rapid sample cleanup: Immunochemical and hplc analysis. Arab. J. Sci. Eng. 2014, 39, 2561-2572.

8. Piletsky, S.A.; Parhometz, Y.P.; Lavryk, N.V.; Panasyuk, T.L.; El'Skaya, A.V. Sensors for low-weight organic molecules based on molecular imprinting technique. Sens. Actuat. B-Chem. 1994, 19, 629-631. [CrossRef]

9. Mirata, F.; Resmini, M. Molecularly Imprinted Polymers for Catalysis and Synthesis; Springer International Publishing: Cham, Switzerland, 2015; pp. 107-129.

10. Norell, M.C.; Andersson, H.S.; Nicholls, I.A. Theophylline molecularly imprinted polymer dissociation kinetics: A novel sustained release drug dosage mechanism. J. Mol. Recognit. 1998, 11, 98-102. [CrossRef]

11. Alvarez-Lorenzo, C.; Concheiro, A. Molecularly imprinted polymers for drug delivery. J. Chromatogr. B 2004, 804, 231-245. [CrossRef] [PubMed]

12. Cunliffe, D.; Kirby, A.; Alexander, C. Molecularly imprinted drug delivery systems. Adv. Drug. Deliv. Rev. 2005, 57, 1836-1853. [CrossRef] [PubMed]

13. Yoshimatsu, K.; Reimhult, K.; Krozer, A.; Mosbach, K.; Sode, K.; Ye, L. Uniform molecularly imprinted microspheres and nanoparticles prepared by precipitation polymerization: The control of particle size suitable for different analytical applications. Anal. Chim. Acta 2007, 584, 112-121. [CrossRef] [PubMed]

14. Cederfur, J.; Pei, Y.X.; Meng, Z.H.; Kempe, M. Synthesis and screening of a molecularly imprinted polymer library targeted for penicilling. J. Comb. Chem. 2003, 5, 67-72. [CrossRef] [PubMed]

15. Wang, J.F.; Cormack, P.A.G.; Sherrington, D.C.; Khoshdel, E. Monodisperse, molecularly imprinted polymer microspheres prepared by precipitation polymerization for affinity separation applications. Angew. Chem. Int. Ed. 2003, 42, 5336-5338. [CrossRef] [PubMed]

16. Hu, S.G.; Li, L.; He, X.W. Comparison of trimethoprim molecularly imprinted polymers in bulk and in sphere as the sorbent for solid-phase extraction and extraction of trimethoprim from human urine and pharmaceutical tablet and their determination by high-performance liquid chromatography. Anal. Chim. Acta 2005, 537, 215-222.

17. Lai, J.P.; Yang, M.L.; Niessner, R.; Knopp, D. Molecularly imprinted microspheres and nanospheres for di(2-ethylhexyl)phthalate prepared by precipitation polymerization. Anal. Bioanal. Chem. 2007, 389, 405-412. [CrossRef] [PubMed]

18. Nagaoka, S.; Ihara, H.; Honbo, J.; Hirayama, C.; Kurisaki, H.; Ikegami, S. Spherical carbon packings prepared from spherical cellulose particles for high-performance liquid chromatography. Anal. Sci. 1994, 10, 543-551. [CrossRef]

19. Wu, N.; Clausen, A.M. Fundamental and practical aspects of ultrahigh pressure liquid chromatography for fast separations. J. Sep. Sci. 2007, 30, 1167-1182. [CrossRef] [PubMed]

20. Meouche, W.; Laatikainen, K.; Margaillan, A.; Silvonen, T.; Siren, H.; Sainio, T.; Beurroies, I.; Denoyel, R.; Branger, C. Effect of porogen solvent on the properties of nickel ion imprinted polymer materials prepared by inverse suspension polymerization. Eur. Polym. J. 2017, 87, 124-135. [CrossRef]

21. Zhang, N.; Hu, X.; Guan, P.; Du, C.; Li, J.; Qian, L.; Zhang, X.; Ding, S.; Li, B. Preparation of protein imprinted microspheres using amphiphilic ionic liquid as stabilizer and emulsifier via miniemulsion polymerization. Biochem. Eng. J. 2017, 317, 356-367. [CrossRef]

22. Guan, X.W.; Li, X.Y.; Chai, S.G.; Zhang, X.H.; Zou, Q.C.; Zhang, J.Z. A sensitive electrochemical sensor based on solution polymerized molecularly imprinted polymers for procaine detection. Electroanalysis 2016, 28, 2007-2015. [CrossRef] 
23. Sambe, H.; Hoshina, K.; Haginaka, J. Molecularly imprinted polymers for triazine herbicides prepared by multi-step swelling and polymerization method: Their application to the determination of methylthiotriazine herbicides in river water. J. Chromatogr. A 2007, 1152, 130-137. [CrossRef] [PubMed]

24. Kan, X.; Zhao, Q.; Zhang, Z.; Wang, Z.; Zhu, J.J. Molecularly imprinted polymers microsphere prepared by precipitation polymerization for hydroquinone recognition. Talanta 2008, 75, 22-26. [CrossRef] [PubMed]

25. Kolb, H.C.; Finn, M.G.; Sharpless, K.B. Click chemistry: Diverse chemical function from a few good reactions. Angew. Chem. Int. Ed. 2001, 40, 2004-2021. [CrossRef]

26. Hizal, G.; Tunca, U.; Sanyal, A. Discrete macromolecular constructs via the diels-alder "click" reaction. J. Polym. Sci. Pol. Chem. 2011, 49, 4103-4120. [CrossRef]

27. Xu, C.; Ye, L. Clickable molecularly imprinted nanoparticles. Chem. Commun. 2011, 47, 6096-6098. [CrossRef] [PubMed]

28. Xu, Z.; Deng, P.; Tang, S.; Kuang, D.; Zhang, F.; Li, J. Preparation of 2d molecularly imprinted materials based on mesoporous silicas via click reaction. J. Mater. Chem. 2014, 2, 8418-8426. [CrossRef]

29. Stephenson-Brown, A.; Acton, A.L.; Preece, J.A.; Fossey, J.S.; Mendes, P.M. Selective glycoprotein detection through covalent templating and allosteric click-imprinting. Chem. Sci. 2015, 6, 5114-5119. [CrossRef]

30. Hou, Y.; Cao, S.; Li, X.; Wang, B.; Pei, Y.; Wang, L.; Pei, Z. One-step synthesis of dual clickable nanospheres via ultrasonic-assisted click polymerization for biological applications. ACS Appl. Mater. Interfaces 2014, 6, 16909-16917. [CrossRef] [PubMed]

31. Hou, Y.; Cao, S.; Wang, L.; Pei, Y.; Zhang, G.; Zhang, S.; Pei, Z. Morphology-controlled dual clickable nanoparticles via ultrasonic- assisted click polymerization. Polym. Chem. 2014, 6, 223-227. [CrossRef]

32. Falk, H. From the photosensitizer hypericin to the photoreceptor stentorin- the chemistry of phenanthroperylene quinones. Angew. Chem. Int. Ed. 1999, 38, 3116-3136. [CrossRef]

33. Pei, Y.-X.; Li, Z.-B.; Pei, Z.-C.; Hou, Y. An Efficient Method for the Synthesis of Hypericin by Monochromatic Light. CN 103274920 A, 17 December 2014.

34. Sun, S.S.; Lees, A.J. Synthesis and photophysical properties of dinuclear organometallic rhenium(i) diimine complexes linked by pyridine-containing macrocyclic: Phenylacetylene ligands. Organometallics 2001, 20, 2353-2358. [CrossRef]

35. Dana, B.H.; Robinson, B.H.; Simpson, J. Intramolecular interactions in 2,6-pyridylacetylenes and their $\mathrm{Co}_{2}(\mathrm{CO})_{4} \mathrm{dppm}$ complexes. J. Organomet. Chem. 2002, 648, 251-269. [CrossRef]

36. Pant, C.S.; Wagh, R.M.; Nair, J.K.; Gore, G.M.; Venugopalan, S. Synthesis and characterization of two potential energetic azido esters. Propellant Explos. Pyrotech. 2006, 31, 477-481. [CrossRef]

37. Jin, F.; Zheng, M.L.; Zhang, M.L.; Zhao, Z.S.; Duan, X.M. A facile layer-by-layer assembly method for the fabrication of fluorescent polymer/quantum dot nanocomposite thin films. RSC Adv. 2014, 4, 33206-33214. [CrossRef]

38. Cheng, W.X.; Fan, F.F.; Zhang, Y.; Pei, Z.C.; Wang, W.J.; Pei, Y.X. A facile approach for fabrication of core-shell magnetic molecularly imprinted nanospheres towards hypericin. Polymers 2017, 9. [CrossRef]

39. Ansell, R.J. Characterization of the Binding Properties of Molecularly Imprinted Polymers. Adv. Biochem. Eng. Biotechnol. 2015, 150, 51-93. [PubMed]

40. Wu, X. Molecular imprinting for anion recognition in aqueous media. Microchim. Acta 2012, 176, $23-47$. [CrossRef]

41. Cheong, W.J.; Yang, S.H.; Ali, F. Molecular imprinted polymers for separation science: A review of reviews. J. Sep. Sci. 2013, 36, 609-628. [CrossRef] [PubMed]

42. Nematollahzadeh, A.; Lindemann, P.; Sun, W.; Stute, J.; Lütkemeyer, D.; Sellergren, B. Robust and selective nano cavities for protein separation: An interpenetrating polymer network modified hierarchically protein imprinted hydrogel. J. Chromatogr. A 2014, 1345, 154-163. [CrossRef] [PubMed]

43. Li, Z.Z.; Qin, C.L.; Li, D.M.; Hou, Y.Z.; Li, S.B.; Sun, J.J. Molecularly imprinted polymer for specific extraction of hypericin from hypericum perforatum 1. Herbal extract. J. Pharm. Biomed. Anal. 2014, 98, 210-220. [CrossRef] [PubMed]

44. Senhadjikebiche, O.; Belaid, T.; Benamor, M. Preparation and characterization of molecularly imprinted polymer as spe sorbent for melamine isolation. Polymers 2013, 5, 1215-1228.

45. Kupai, J.; Razali, M.; Buyuktiryaki, S.; Kecili, R.; Szekely, G. Long-term stability and reusability of molecularly imprinted polymers. Polym. Chem. 2017, 8, 666-673. [CrossRef] [PubMed] 
(C) 2017 by the authors. Licensee MDPI, Basel, Switzerland. This article is an open access article distributed under the terms and conditions of the Creative Commons Attribution (CC BY) license (http:/ / creativecommons.org/licenses/by/4.0/). 